\section{Agreement of non-contact pachymetry after LASIK: comparison of combined scanning-slit/Placido disc topography and specular microscopy}

A López-Miguel'1 JC Nieto', M Díez-Cuenca', DP Piñero ${ }^{2}$ and MJ Maldonado ${ }^{1,3}$
${ }^{1}$ Departamento de Oftalmología, Clínica Universitaria, Universidad de Navarra, Pamplona, Spain

${ }^{2}$ Vissum-Instituto Oftalmológico de Alicante, Universidad Miguel Hernández, Alicante, Spain

${ }^{3}$ IOBA, University of Valladolid, Valladolid, Spain

Correspondence: A López-Miguel, Departamento de Oftalmología, Clínica Universitaria, Av. Pío XII, 36, Pamplona 31008, Spain Tel: + 34948296331 ; Fax: + 34948296500 . E-mail: alberoptico@ yahoo.es

Received: 8 May 2009 Accepted in revised form: 19 August 2009 Published online: 25 September 2009

\begin{abstract}
Purpose To assess the interchangeability of central corneal thickness (CCT) measurements between combined scanning-slit/Placido disc topography (Orbscan-II) and specular microscopy (Topcon SP-2000P) in patients who underwent laser in situ keratomileusis (LASIK) for myopia.

Patients and methods We recruited 118 consecutive patients who underwent LASIK for myopia. The CCT was measured using Orbscan-II and Topcon SP-2000P randomly. Orbscan-II and Topcon SP-2000P CCT data were analysed using the paired-sample $t$-test and the limits of agreement (LoA) were calculated with the method described by Bland-Altman.

Results The average CCT measurements by Orbscan-II and Topcon SP-2000P were $447.55 \pm 49.78 \mu \mathrm{m}$ and $461.38 \pm 35.35 \mu \mathrm{m}$, respectively $(P<0.0001$; mean difference, $13.83 \pm 22.31 \mu \mathrm{m} ; \mathbf{9 5} \%$ confidence interval, 9.77-17.09 $\mu \mathrm{m})$. The Bland-Altman plot showed an inverse association between the average and the difference between the devices: TopconOrbscan-II $=174.23-0.353 \times$ Average $(P<0.01)$. The widths of the crude and regression-based 95\% limits of agreement were 87.45 and $63.72 \mu \mathrm{m}$, respectively.

Conclusions Orbscan-II measurements of CCT after myopic LASIK were significantly lower than those obtained using Topcon SP-2000P. The limits of agreement between the two devices were too broad and, therefore, both pachymetric values cannot be used interchangeably. Further, the tendency
\end{abstract}

towards comparably Orbscan-II readings in thinner corneas precludes that one technique can directly replace the other. This is important for the adequate medium- and longterm follow-up of the growing LASIK patient population.

Eye (2010) 24, 1064-1070; doi:10.1038/eye.2009.233; published online 25 September 2009

Keywords: central corneal thickness; specular microscopic pachymetry; Orbscan-II pachymetry; LASIK

The measurement of central corneal thickness (CCT) is clinically relevant, especially considering the increasing popularity of refractive surgery. Accurate analysis of the corneal thickness is of paramount importance for assessing corneal disease and non-refractive and refractive surgical procedures. ${ }^{1,2}$

Measurement errors could affect the preoperative assessment of candidates for primary and retreatment laser in situ keratomileusis (LASIK), ${ }^{3-6}$ the intraocular lens (IOL) power calculations after LASIK, $^{7}$ and the contribution of the CCT to measurement of the intraocular pressure (IOP) ${ }^{8}$

CCT can be measured using various techniques. Ultrasonic pachymetry is the current gold standard for pachymetry measurement, ${ }^{9,10}$ but ultrasonography requires a probe contact. Thus, direct corneal trauma might occur resulting in flap dislocation, ${ }^{11}$ which also might occur with a history of minor trauma; ${ }^{12}$ the risk of microbial contamination is associated with use of a probe, ${ }^{13}$ and topical 
anaesthesia must be instilled. All of these factors might be associated with patient discomfort. Moreover, pachymetry measurements might be erroneous because of epithelial indentation; the accuracy will depend on the exact axial placement of the probe relative to the centre of the cornea. ${ }^{14}$ Therefore, non-contact methods are preferred for ocular biometry, and alternative techniques such as interferometry, ${ }^{15}$ corneal confocal microscopy, ${ }^{16}$ optical coherence tomography, ${ }^{9}$ non-contact specular microscopy, ${ }^{17}$ and scanning-slit technology $y^{5}$ are gaining popularity for assessing patients who have undergone LASIK.

In particular, the Orbscan-II (Bausch \& Lomb, Rochester, NY, USA) topography system provides widefield pachymetry analysis of the anterior and posterior corneal elevations. The corneal thickness can be calculated by measuring the distance between the anterior and posterior corneal surfaces. ${ }^{18}$

The Topcon non-contact specular microscope (NCSM) (SP-2000P; Topcon Corp., Tokyo, Japan) provides pachymetry measurements and specular microscopy simultaneously. ${ }^{14}$ Focusing on the endothelium, this apparatus supplies specular images and measures the focal distance, which can be calculated as the corneal thickness. ${ }^{19}$

Many ophthalmologists rely on the results yielded by both non-contact devices. Despite both non-contact methods are convenient, no study has analysed their interchangeability after myopic LASIK properly. The only study that compared both used correlation coefficients to assess their relationship, ${ }^{3}$ an analysing method that is flawed based on the investigations of Bland and Altman, ${ }^{20,21}$ as well as the model of regression that relates the measurements obtained by both devices. Therefore, we evaluated whether the devices are interchangeable for patients who had undergone LASIK using the appropriate methods described by Bland and Altman. ${ }^{20,21}$

\section{Materials and methods}

This study adhered to the principles outlined in the Declaration of Helsinki Principles. All candidates received detailed information about the nature of the investigation, and all provided informed consent. We selected 118 consecutive patients who had complete refractive stability on two successive visits at least 2 months apart. One eye of each patient was selected randomly for this investigation and the measurements were taken 9 months after LASIK. Patients were recruited over a 2-year period. Exclusion criteria consisted of suspected keratectasia, progressive myopia, or astigmatism (patients with a 0.25-diopter [D] change and/or $\geqslant 15$ degrees in cyclopegic refractive cylinder orientation were excluded); preoperative or post-LASIK hyperopia; mixed astigmatism; active ocular disease; connective tissue disorders; and pregnancy. All eyes underwent a complete ophthalmic examination before and after LASIK, including manifest and cycloplegic refractions, Orbscan-II topography, Topcon NCSM pachymetry, slit-lamp microscopy, applanation tonometry, and indirect ophthalmoscopy. Objective measurement of the corneal transparency was performed as previously reported. ${ }^{22}$

One experienced technician was assigned to each instrument to minimize variations in the results. The examiner was masked to the refraction and the postoperative time interval at each examination. Central pachymetry also was measured using the Topcon NCSM and compared with the Orbscan-II pachymetry values. The testing sequence of the measurements with both devices was randomized to avoid methodological and statistical bias. ${ }^{23}$ Examinations were carried out from 11:00 am to 1:00 pm to minimize the effect of diurnal variations on corneal thickness. ${ }^{24}$ Non-contact pachymetry measurements were always taken prior to any other ophthalmic procedure that could alter the cornea, including applanation tonometry.

\section{Surgical procedures}

All procedures were performed by the same surgeon (MJ Maldonado). The details of the primary LASIK procedures have been reported previously. ${ }^{6}$ The preoperative CCT had to be adequate to ensure a residual stromal bed of at least $250 \mu \mathrm{m}$ and above $55 \%$ of the total preoperative CCT after creation of a corneal flap with a superior hinge $(160-\mu \mathrm{m}$ head, Hansatome microkeratome; Chiron Vision, Claremont, CA, USA). Ablation of the appropriate cut depth was carried out using the Technolas Keracor 217z excimer laser (Bausch \& Lomb Chiron Technolas GmbH, Munich, Germany).

\section{Topographic evaluation of CCT}

Orbscan-II was calibrated before each session test following the instructions of the operator's manual provided by the manufacturer. ${ }^{25}$ The patient was instructed to fixate on a flickering red light and to blink to obtain a homogeneous tear film layer that ensures more reliable results. The instrument was then aligned and the cornea was scanned using a slit beam. The Orbscan-II statistical analysis device (software version 3.12) calculated the elevation of the anterior and posterior corneal surfaces; thus, the pachymetry measurement is the result of subtraction of the elevation maps of both corneal surfaces. Although Orbscan-II displays pachymetry values at several locations, only the 
central pachymetry value, but not the thinnest corneal point, was taken into account for statistical analysis because of two reasons: first, the reproducibility of the thinnest corneal point is expected to be lower ${ }^{26}$ and second, Topcon NCSM does not provide the thinnest corneal value, thus precluding comparison between measurement methods. The default acoustic equivalent setting of 0.95 for software version $3.12^{27}$ was used throughout this study.

\section{Non-contact specular microscopy evaluation of the CCT}

Topcon NCSM was calibrated as previously described..$^{28}$ The CCT was measured while the subject fixated on a fixation light in the specular microscope. The pachymetry of the central cornea was obtained after proper positioning of the alignment dot, square, and bar on the screen using the automatic mode. The automated image capture, low-intensity mode of the specular microscope was used as described previously. ${ }^{17}$ The CCT was determined by patient fixation as well as Orbscan-II examination.

\section{Statistical analyses}

Data from the prospectively completed forms were entered into a database and statistical calculations were performed using SPSS (version 15.0 for Windows). The mean and standard deviation (SD) were calculated for normally distributed data.

Where data did not correspond to a normal distribution, the median (50th percentile) and interquartile range (IR: values between the 25 th and 75 th percentiles of the distribution) were used instead. For all statistical tests, a two-tailed $P<0.05$ was considered significant. The paired $t$-test was used to establish whether or not there was a significant systematic bias between measurements.

Bland-Altman graphs of the differences against the means were plotted to check the relation between the differences and the range of measurement, and to check whether the differences between the measurements were approximately normally distributed..$^{20,21}$ We assessed the interchangeability of both measurement methods by calculating the $95 \%$ limits of agreement (LoA), defined as the mean difference in measurements $\pm 1.96 \mathrm{SD}$ as suggested by Bland and Altman. ${ }^{20,21}$

When the differences between methods of measurement tend to be in one direction for low values of the quantity being measured and in the other direction for high values, a better fit than the above-mentioned crude LoA can be found by using a regression model described by Bland and Altman. ${ }^{29}$ A regression of the difference between measurement methods on their average can be used to model the relationship between mean difference and the magnitude being measured. In a second stage of the analysis, modelling the variability in the SD of the differences, directly as a function of the level of the measurement, can be achieved using a method based on absolute residuals from a fitted regression line. Modelling is considerably simplified by the assumption that these residuals have a normal distribution. Once one can predict the mean difference and the SD from these calculations, mean \pm 1.96 SD can be estimated for any magnitude, and can be obtained by combining the two regression equations.

We certify that all applicable institutional and governmental regulations concerning the ethical use of human volunteers were followed during this research. This study was approved by the University of Navarra Clinic Review Committee.

\section{Results}

The average CCT measurements by Orbscan-II and Topcon NCSM were $447.55 \pm 49.78 \mu \mathrm{m}$ (range, 343$548 \mu \mathrm{m}$ ) and $461.38 \pm 35.35 \mu \mathrm{m}$ (range, 392-540 $\mu \mathrm{m}$ ), respectively, a difference that reached statistical significance $(P<0.0001)$. The Orbscan-II readings were lower than those of the Topcon NCSM by an average of $13.83 \pm 22.31 \mu \mathrm{m}$ (95\% confidence interval, 9.77$17.09 \mu \mathrm{m})$. Corneal opacification was almost non-existent (median and IR, 0 gray levels (range, 0-3)).

A Bland-Altman plot, which was created to assess the difference in individual measurement as a function of the mean of two measurements (Figure 1a), revealed poor agreement due to a systematic bias, and the crude LoA were also too wide $(87.45 \mu \mathrm{m}$; range: $-29.89 \mu \mathrm{m}$ to $57.53 \mu \mathrm{m}$ ). Nevertheless, the distribution of the difference between methods was approximately normal (Figure 1b).

We also found an inverse association between the average of both measurements and the difference between Topcon NCSM and Orbscan-II for CCT measurements (Figure 2a); the regression of difference on average gave a significant relationship $(P<0.01)$ :

Difference $($ Topcon NCSM - Orbscan II $)=174.23-0.353 \times$ Average

Therefore, there was a trend in the bias, a tendency for the mean difference to decrease with increasing magnitude. The regression-based 95\% LoA:

$$
\begin{aligned}
& \text { Upper limit }=206.11-0.351 \times \text { Average } \\
& \text { Lower limit }=142.39-0.355 \times \text { Average }
\end{aligned}
$$

are shown in Figure 2a and exhibit almost coincident significant slopes $(0.351$ and 0.355 , both $P<0.01)$. The residuals from the regression of difference on average had a normal distribution (Figure 2b), which indicates 
a

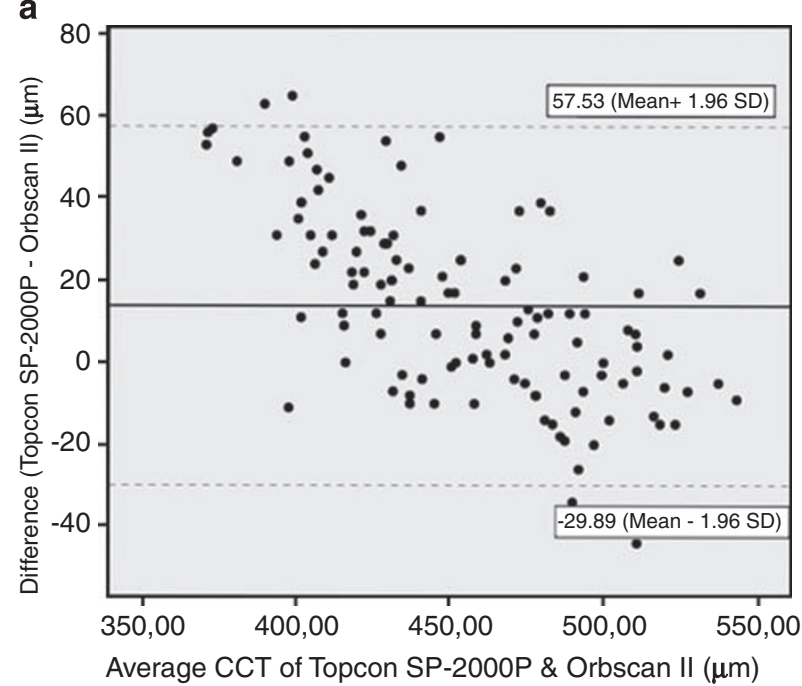

b

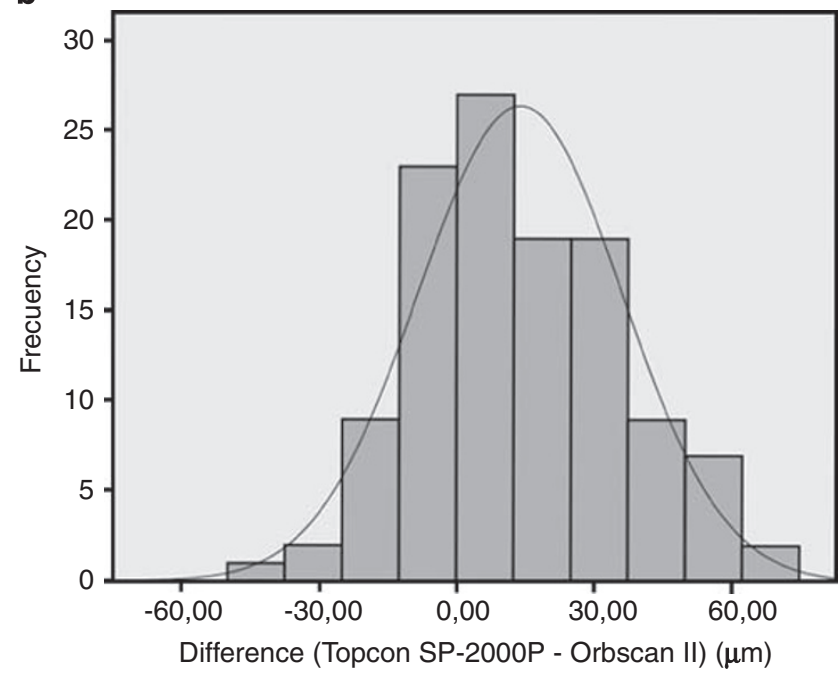

Figure 1 (a) Bland-Altman plot comparing the two modalities. The solid line represents the mean difference in CCT values and the dashed lines represent the crude 95\% confidence limits. (b) Histogram shows the distribution of differences between Topcon NCSM and Orbscan-II CCT measurements.
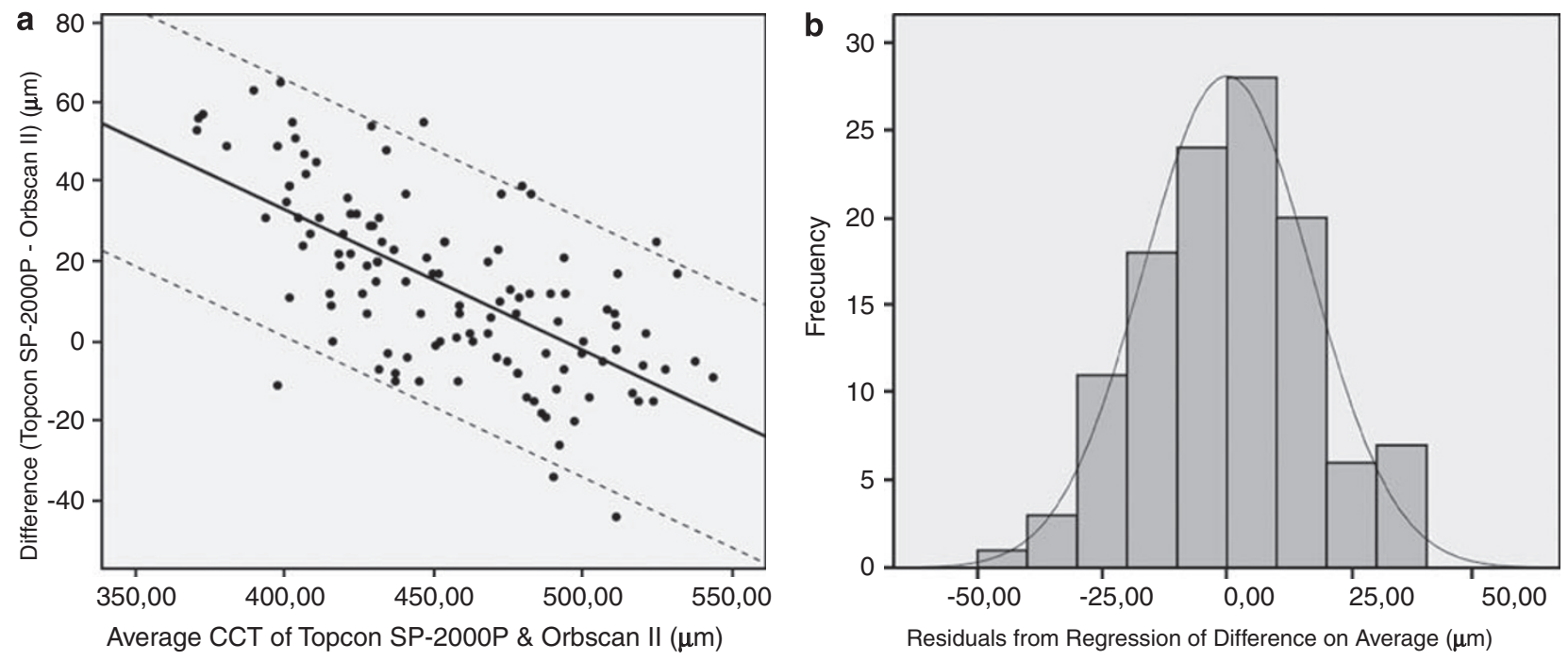

Figure 2 (a) Regression-based LoA for difference in central pachymetry determined by both measurement methods. The solid line represents the regression-based mean difference in CCT values and the dashed line represents the regression-based $95 \%$ confidence limits. (b) Histogram shows the distribution of the residuals from the regression of the differences between Topcon NCSM and Orbscan-II CCT measurements on their average.

the suitability of this method for this data set. The regression-based $95 \%$ LoA were also rather wide (63.72 $\mu \mathrm{m}$; range: $\pm 31.86 \mu \mathrm{m})$.

\section{Discussion}

An accurate assessment of the CCT is essential in several clinical situations, especially for refractive surgery. LASIK currently is the most popular technique; thus, precise corneal thickness measurements are mandatory to reduce the risk of complications related to refractive surgery. Erroneous pachymetry measurements might mislead the clinical decision making regarding LASIK enhancement, because retreatment can be performed only when biomechanical corneal stability has been confirmed and corneal thickness allows for further ablation to avoid keratectasia. ${ }^{6}$ Measurement errors also can result in miscalculation of the IOL power in patients 
who have undergone $\mathrm{LASIK}^{7}$ and might affect the screening of patients with glaucoma due to a reduced IOP measurement. ${ }^{8}$

Although ultrasound pachymetry is the most commonly used method in clinical practice, access to accurate non-invasive pachymetry is important to reduce the risk of corneal epithelial damage or flap displacement resulting from probe contact, and to avoid the risk of microbial contamination during follow-up of patients after LASIK. 2,4,13 Therefore, non-contact methods have been developed to avoid these complications.

In the current study, the results of non-contact pachymetry showed that on average Orbscan-II provides lower CCT values than Topcon NCSM in patients who have undergone myopic LASIK. Our results agree with those obtained by Kawana et $a l^{3}$ which also showed Orbscan-II pachymetric underestimation by $22.3 \mu \mathrm{m}$ as compared with Topcon NCSM after LASIK. In the current study, Bland-Altman plots showed that OrbscanII tends to underestimate CCT as compared with Topcon NCSM, which is defined as a systematic bias. This bias in measuring CCT using different acquisition methods in healthy unoperated corneas was reported previously; Tam and Rootman ${ }^{30}$ compared CCT measurements using specular microscopy and ultrasound and reported a systematic bias towards higher CCT values using specular microscopy. Similar underestimation was also described when pathologically thinned corneas in keratoconus patients were measured using different acquisition techniques, scanning-slit/Placido disc topography and Topcon NCSM. ${ }^{14}$

In our study, the Bland-Altman plot also showed a downward trend of the scatter plot; therefore, there is a tendency for the mean difference to fall with higher magnitudes when the Topcon NCSM and Orbscan-II CCT measurements are compared. Although the regression-based LoA improved the data fit in comparison with the crude LoA, which ignored this relationship, both 95\% LoA were too wide (63.72 and $87.45 \mu \mathrm{m})$ for the two pachymetric methods to be considered interchangeable. Chakrabarti et $a l^{2}$ and Cheng et $a l^{31}$ also reported such a tendency when scanning-slit and ultrasound techniques were compared after LASIK. This type of bias was also seen in normal corneas when Amano $e t a l^{32}$ studied the interchangeability of scanningslit and ultrasound pachymetric methods. They found that Orbscan-II tended to underestimate lower CCT values and overestimate higher CCT values, although the difference was not significant.

The discrepancy in measurements with Orbscan-II and Topcon NCSM after LASIK might be attributed to their distinct methods of acquiring data. This hypothesis about the discrepancy in measurements might be similar to those in other studies that compared Orbscan-II pachymetry with ultrasound pachymetry, which also showed Orbscan-II underestimation in patients who underwent LASIK. ${ }^{4,5}$ The underestimation of Orbscan-II pachymetry measurements might be associated with alterations in the postoperative optical quality of the cornea. ${ }^{4,33,34}$ A significant correlation has been established between the grade of corneal transparency loss and the decrease in Orbscan-II pachymetry readings. ${ }^{33}$ However, in our series of patients assessed 9 months after surgery, the corneal transparency was comparable to that of a normal cornea. Although no patients had noticeable post-LASIK haze during the slitlamp examination, confocal microscopy analysis shows increased cell density in LASIK patients, ${ }^{5}$ which produces loss of transparency. This loss increases the reflectivity in the interface with a consequent systematic measurement error. ${ }^{5}$ Another theory suggests that media opacities might bend the light rays passing through the cornea and cause displacement of the slit emitted by Orbscan-II, producing an inaccurate posterior corneal profile. ${ }^{4}$ Another hypothesis suggested that in ablated corneas the newly formed type-III collagen and proteoglycan debris-filled vacuoles resulted in a high level of light scatter, with an ensuing increase in the corneal refraction index. ${ }^{34}$ All authors agreed that loss of corneal transparency progressively decreases with time, thus improving the accuracy of Orbscan-II. ${ }^{4,5}$

Another source of Orbscan-II pachymetry underestimation might be the change in corneal shape after LASIK. The normal healthy cornea is usually prolate, but after myopic LASIK the corneal curvature is altered and tends to become oblate. ${ }^{2}$ The reconstruction algorithms, using lower order polynomials, which create elevation data in the Orbscan-II may be properly designed in a normal corneal surface, but they might be ineffective in a post-LASIK corneal surface. ${ }^{2}$

The main limitation of the current study is that the reason for the variability between measurements showed a wide distribution between methods was not completely addressed, although it can be explained by the measurement repeatability of Orbscan-II being generally lower than that of Topcon NCSM in postmyopic LASIK patients. ${ }^{35}$ In addition, this study included only patients who had undergone LASIK to treat myopia, and, therefore, the conclusions cannot be applied to patients with hyperopia, in whom the postoperative corneal shape is more prolate. Finally, ultrasonic pachymetry was not performed in the study eyes, thus precluding direct comparison of both non-contact methods with the gold standard in order to find out which of the two is closer to the 'true' value. However, such a comparison has already been extensively reported, 2,3,5,10,27,31,36,37 and has been included in the discussion of our results with those of previously published studies. 
In conclusion, these non-contact methods are not interchangeable in patients who underwent LASIK for myopia, because there is a systematic bias between acquisition methods and there is also a tendency for the mean difference to decrease with increasing magnitude. Thus, although the devices were calibrated appropriately, an acoustic factor was changed ${ }^{10}$ or a subtraction method applied, ${ }^{36}$ one cannot reliably replace the other. A tendency and a systematic bias also were described when each scanning-slit and specular pachymetry, respectively, were previously compared with ultrasonic pachymetry in patients who underwent LASIK. ${ }^{31,37}$ Therefore, ophthalmologists should be aware that not only contact and non-contact pachymetry are not interchangeable after LASIK, but also that these non-contact pachymetry methods cannot be used interchangeably in these patients. This is important for the adequate medium- and long-term follow-up of the growing LASIK patient population.

\section{Summary}

\section{What was known before}

- Central corneal thickness measurements of Orbscan-II were significantly smaller than those of non-contact specular microscopy (Topcon SP-2000P) after myopic LASIK, although these instruments were analysed using linear correlation to assess interchangeability, a method that is flawed based on the investigations of Bland and Altman.

\section{What this study adds}

- The limits of agreement of central corneal thickness between combined scanning-slit/Placido disc topography and non-contact specular microscopy were too wide and we detected an association between the magnitude and the difference. Thus both techniques cannot be used interchangeably after myopic LASIK.

\section{Conflict of interest}

The authors declare no conflict of interest

\section{Acknowledgements}

This work was supported in part by RETICS RD07/0062 (Oftalmología) and the Spanish Ministry of Education and Science through research project FIS2005-05020-C03-03.

\section{References}

1 Ghergel D, Hosking SL, Mantry S, Banerjee S, Naro SA, Shah S. Corneal pachymetry in normal and keratoconic eyes: Orbscan II versus ultrasound. J Cataract Refract Surg 2004; 30: 1272-1277.
2 Chakrabarti HS, Craig JP, Brahma A, Malik TY, McGhee CN. Comparison of corneal thickness measurements using ultrasound and Orbscan slit-scanning topography in normal and post-LASIK eyes. J Cataract Refract Surg 2001; 27: 1823-1828.

3 Kawana K, Tokunaga T, Miyata K, Okamoto F, Kiuchi T, Oshika T. Comparison of corneal thickness measurements using Orbscan II, non-contact specular microscopy, and ultrasonic pachymetry in eyes after laser in situ keratomileusis. Br J Ophthalmol 2004; 88: 466-468.

4 Prisant O, Calderon N, Chastang P, Gatinel D, Hoang-Xuan T. Reliability of pachymetric measurements using Orbscan after excimer refractive surgery. Ophthalmology 2003; 110: 511-515.

5 Cheng AC, Rao SK, Tang E, Lam DS. Pachymetry assessment with Orbscan II in postoperative patients with myopic LASIK. J Refract Surg 2006; 22: 363-366.

6 Maldonado MJ. Undersurface ablation of the flap for laser in situ keratomileusis retreatment. Ophthalmology 2002; 109: 1453-1464.

7 Hamed AM, Wang L, Misra M, Koch DD. A comparative analysis of five methods of determining corneal refractive power in eyes that have undergone myopic laser in situ keratomileusis. Ophthalmology 2002; 109: 651-658.

8 Emara B, Probst LE, Tingey DP, Kennedy DW, Willms LJ, Machat J. Correlation of intraocular pressure and central corneal thickness in normal myopic eyes and after laser in situ keratomileusis. J Cataract Refract Surg 1998; 24: 1320-1325.

9 Maldonado MJ, Ruiz-Oblitas L, Munuera JM, Aliseda D, Garcia-Layana A, Moreno-Montañés J. Optical coherence tomography evaluation of the corneal cap and stromal bed features after laser in situ keratomileusis for high myopia and astigmatism. Ophthalmology 2000; 107: 81-87.

10 Ho T, Cheng AC, Rao SK, Lau S, Leung CK, Lam DS. Central corneal thickness measurements using Orbscan II, Visante, ultrasound, and Pentacam pachymetry after laser in situ keratomileusis for myopia. J Cataract Refract Surg 2007; 33: 1177-1182.

11 Maldonado MJ, Juberias JR. Subtarsal flap dislocation after superior hinge laser in situ keratomileusis in a patient with borderline mental illness. J Refract Surg 2003; 19: 169-171.

12 Lombardo AJ, Katz HR. Late partial dislocation of a laser in situ keratomileusis flap. J Cataract Refract Surg 2001; 27: 1108-1110.

13 Maldonado MJ. Corneal epithelial alterations resulting from use of chlorine-disinfected contact tonometer after myopic photorefractive keratectomy. Ophthalmology 1998; 105: 1546-1549.

14 Kawana K, Miyata K, Tokunaga T, Kiuchi T, Hiraoka T, Oshika T. Central corneal thickness measurements using Orbscan II scanning slit topography, noncontact specular microscopy, and ultrasonic pachymetry in eyes with keratoconus. Cornea 2005; 24: 967-971.

15 Hitzenberger CK, Baumgartner A, Drexler W, Fercher AF Interferometric measurement of corneal thickness with micrometer precision. Am J Ophthalmol 1994; 118: 468-476.

16 Brugin E, Ghirlando A, Gambato C, Midena E. Central corneal thickness: z-ring corneal confocal microscopy versus ultrasound pachymetry. Cornea 2007; 26: 303-307.

17 Ogbuehi KC, Almubrad TM. Repeatability of central corneal thickness measurements measured with the Topcon SP2000P specular microscope. Graefes Arch Clin Exp Ophthalmol 2005; 243: 798-802. 
18 Cairns G, McGhee CN. Orbscan computerized topography: attributes, applications, and limitations. J Cataract Refract Surg 2005; 31: 205-220.

19 Módis Jr L, Langenbucher A, Seitz B. Scanning-slit and specular microscopic pachymetry in comparison with ultrasonic determination of corneal thickness. Cornea 2001; 20: 711-714.

20 Bland JM, Altman DG. Statistical methods for assessing agreement between two methods of clinical measurement. Lancet 1986; 1: 307-310.

21 Altman DG, Bland JM. Measurement in medicine: the analysis of method comparison studies. The Statistician 1983; 32: 307-317.

22 Maldonado MJ, Arnau V, Martínez-Costa R, Navea A, Mico FM, Cisneros AL et al. Reproducibility of digital image analysis for measuring corneal haze after myopic photorefractive keratectomy. Am J Ophthalmol 1997; 123: 31-41.

23 Fisher LD, Van Belle G. Biostatistics: a Methodology for the Health Sciences. Wiley Intersciences: New York, 1993.

24 Lattimore Jr MR, Kaupp S, Schallhorn S, Lewis IV R. Orbscan pachymetry: implications of a repeated measures and diurnal variation analysis. Ophthalmology 1999; 106: 977-981.

25 Bausch and Lomb. Orbscan II, Orbscan IIZ, Operator's Manual, Version 3.12. Bausch \& Lomb: Salt Lake City, Utah, 2002

26 Fam HB, Lim KL, Reinstein DZ. Orbscan global pachymetry: analysis of repeated measures. Optom Vis Sci 2005; 82: 1047-1053.

27 Myrowitz EH, Ren S, Chuck RS. Comparison of central corneal thickness measured by four different pachymeters. Eye Contact Lens 2007; 33: 156-160.

28 Van Shaick W, van Dooren BT, Malder PG, Völker-Dieben HJ. Validity of endothelial cell analysis methods and recommendations for calibration in Topcon SP-2000P specular microscopy. Cornea 2005; 24: 538-544.

29 Bland JM, Altman DG. Measuring agreement in method comparison studies. Stat Methods Med Res 1999; 8: 135-160.

30 Tam ES, Rootman DS. Comparison of central corneal thickness measurements by specular microscopy, ultrasound pachymetry, and ultrasound biomicroscopy. J Cataract Refract Surg 2003; 29: 1179-1184.

31 Cheng AC, Rao SK, Lau S, Leung CK, Lam DS. Central corneal thickness measurements by ultrasound, Orbscan II, and Visante OCT after LASIK for myopia. J Refract Surg 2008; 24: 361-365.

32 Amano S, Honda N, Amano Y, Yamagami S, Miyai T, Samejima $\mathrm{T}$ et al. Comparison of central corneal thickness measurements by rotating Scheimpflug camera, ultrasonic pachymetry, and scanning-slit corneal topography. Ophthalmology 2006; 113: 937-941.

33 Fakhry MA, Artola A, Belda JI. Comparison of corneal pachymetry using ultrasound and Orbscan II. J Cataract Refract Surg 2002; 28: 248-252.

34 Boscia F, La Tegola MG, Alessio G, Sborgia C. Accuracy of Orbscan optical pachymetry in corneas with haze. J Cataract Refract Surg 2002; 28: 253-258.

35 Maldonado MJ, Lopez-Miguel A, Nieto JC, Cano-Parra J, Calvo B, Alio JL. Reliability of noncontact pachymetry after laser in situ keratomileusis. Invest Ophthalmol Vis Sci 2009; 50: 4135-4141.

36 Cheng AC, Tang E, Mohamed S, Lam DS. Correction factor in Orbscan II in the assessment of corneal pachymetry. Cornea 2006; 25: 1158-1161.

37 Zhao MH, Zou J, Wang WQ, Li J. Comparison of central corneal thickness as measured by non-contact specular microscopy and ultrasound pachymetry before and post LASIK. Clin Exp Ophthalmol 2007; 35: 818-823. 